Volume 140, Number 12, December 2012, Pages 4111-4126

S 0002-9939(2012)11583-7

Article electronically published on April 12, 2012

\title{
QUADRATIC TRANSFORMS INSIDE THEIR GENERIC INCARNATIONS
}

\author{
SHREERAM S. ABHYANKAR \\ (Communicated by Lev Borisov)
}

\begin{abstract}
The generic incarnation of a quadratic transform is obtained by adjoining an indeterminate. This leads to the singularity tree of a generic member of a pencil of curves.
\end{abstract}

\section{INTRODUCTION}

Given any two-dimensional regular local domain $R$, let $Q(R)$ be the set of all two-dimensional iterated quadratic transforms of $R$, and let $L$ be the quotient field of $R$. For the terminology to be used in this paper, see Section 2 and Remark (4.0) of [Ab11] for further notation and terminology, see Section 1 of my book [Ab3] and pages 146-161 and 552-577 of my book Ab8]; more background material can be found in $\mathrm{Ab} 9, \mathrm{Ab} 10, \mathrm{Ab} 12$. By $\mathrm{Ab1}$ (also see $\mathrm{Ab} 2$ ) $Q(R)$ coincides with the set of all two-dimensional regular local domains whose quotient field is $L$ and which dominate $R$. Consider the rational function field $L(t)$ in an indeterminate $t$ over $L$. For every subring $S$ of $L$ let $S^{t}$ be the localization of $S[t]$ at the multiplicative set of all those members of $S[t]$ whose coefficients generate the unit ideal in $S$. Let $Q^{t}(R)=\left\{S^{t}: S \in Q(R)\right\}$. We shall show that $Q^{t}(R) \subset Q\left(R^{t}\right)$. We may refer to $Q^{t}(R)$ as the generic incarnation of $Q(R)$. Given any nonzero element $F$ in the maximal ideal $M(R)$ of $R$, we visualize $R$ as the local ring of a simple point $P$ of an algebraic or arithmetic surface and $F=0$ as the local equation of a curve $C$ on the surface passing through $P$. Now the singularity tree of $C$ at $P$ is a subset of $Q(R)$. For singularity trees, see Figure 5 on page 426 of [Ab4], Figure 18.2 on page 132 of Ab6], and the material of [Ab5]. Let $G=0$ be the local equation of a curve having no common component with $F=0$ at $P$. We are interested in the singularity tree of a generic member of the pencil generated by $F$ and $G$. As a concrete illustration, take $R$ to be the localization of the bivariate polynomial ring $k[X, Y]$ over a field $k$ at the maximal ideal generated by $(X, Y)$, and take $F$ and $G$ to be nonconstant polynomials $F(X, Y)$ and $G(X, Y)$ having no nonconstant common factor. Now $R^{t}$ is the localization of $k(t)[X, Y]$ at the maximal ideal generated by $(X, Y)$ and we let $\Phi=\Phi(X, Y)=F(X, Y)+t G(X, Y) \in k(t)[X, Y]$. Then the local equation of a generic member of the pencil generated by $F$ and $G$ is $\Phi=0$, and we study its singularity tree. Elsewhere we shall relate this tree to the dicritical divisors of the said pencil.

Received by the editors May 25, 2011.

2010 Mathematics Subject Classification. Primary 14A05.

Key words and phrases. Pencil, singularity tree, generic member.

(C) 2012 American Mathematical Society Reverts to public domain 28 years from publication 


\section{TERMinOLOGY}

Consider the rational function field $L(t)$ in an indeterminate $t$ over a field $L$. We define the $t$-extension $S^{t}$ of any subring $S$ of $L$ by putting

$$
S^{t}=\left\{\begin{array}{l}
\text { the localization of the polynomial ring } S[t] \\
\text { at the multiplicative set of all those members of } S[t] \\
\text { whose coefficients generate the unit ideal in } S .
\end{array}\right.
$$

Note that $L^{t}=L(t)$ and, in Nagata's notation (page 18 of $\mathrm{Nag}$ ), $S^{t}=S(t)$. Clearly

$$
\left\{\begin{array}{l}
S \mapsto S^{t} \text { gives a map } \rho^{t}: \rho(L) \rightarrow \rho\left(L^{t}\right), \text { where } \rho(L) \text { and } \rho\left(L^{t}\right) \\
\text { are the sets of all subrings of } L \text { and } L^{t} \text { respectively. }
\end{array}\right.
$$

To supplement Section 2 of Ab11] we introduce some more terms. So given any two-dimensional regular local domain $R$ we put:

$D_{j}(R)^{\Delta}=\left\{o(S): S \in Q_{j}(R)\right\}=j$-th layer of the set of all prime divisors of $R$.

$D(R)^{\Delta}=\coprod_{j \in \mathbb{N}} D_{j}(R)^{\Delta}=$ set of all prime divisors of $R$.

$Q_{j}(R)=j$-th layer of the quadratic tree of $R$.

$Q(R)=\coprod_{j \in \mathbb{N}} Q_{j}(R)=$ the quadratic tree of $R$.

$Q(R)_{j}^{\Delta}=Q_{j}(R) \amalg D(R)_{j}^{\Delta}=j$-th layer of the full quadratic tree of $R$.

$Q(R)^{\Delta}=\coprod_{j \in \mathbb{N}} Q(R)_{j}^{\Delta}=$ the full quadratic tree of $R$.

$R^{t}=t$-extension of $R$.

$Q_{j}^{t}(R)=\left\{S^{t}: S \in Q_{j}(R)\right\}=j$-th layer of $t$-extension of the quadratic tree of $R$.

$Q^{t}(R)=\left\{S^{t}: S \in Q(R)\right\}=\coprod_{j \in \in \mathbb{N}} Q_{j}^{t}(R)=t$-extension of the quadratic tree of $R$.

Note that $Q^{t}(R)$ as well as its complement in $Q\left(R^{t}\right)$ are both infinite sets. They are sort of analogues of rational and irrational points of the real line. Actually they are more analogues to the $k$-algebraic and $k$-transcendental points of the projective line over $k(t)$, where $k$ is a subfield of $L$. At any rate, the said two infinite sets have the same cardinality.

In Section 2 of Ab11, for any $T \in Q(R)$ and any nonzero ideal $I$ in $R$, we have defined the concept of the $(R, T)$-transform of $I$, which we denote by $(R, T)(I)$. Now we define:

$\mathfrak{Q}_{j}(R, I)=\left\{T \in Q_{j}(R):(R, T)(I)\right.$ is not principal $\}=j$-th layer of the ideal tree of $I$ in $R$,

$\mathfrak{Q}(R, I)=\{T \in Q(R):(R, T)(I)$ is not principal $\}=\coprod_{j \in \mathbb{N}} \mathfrak{Q}_{j}(R, I)=$ the ideal tree of $I$ in $R$,

$\mathfrak{P}_{j}(R, I)=\left\{T \in Q_{j}(R):(R, T)(I) \neq T\right\}=j$-th layer of the singularity tree of $I$ in $R$,

$\mathfrak{P}(R, I)=\{T \in Q(R):(R, T)(I) \neq T\}=\coprod_{j \in \mathbb{N}} \mathfrak{P}_{j}(R, I)=$ the singularity tree of $I$ in $R$,

and for any nonzero element $z$ in the quotient field of $R$ we define: $\mathfrak{Q}_{j}(R, z)=\mathfrak{Q}_{j}\left(R, J_{R}(z)\right)=j$-th layer of the ideal tree of $z$ in $R$, $\mathfrak{Q}(R, z)=\mathfrak{Q}\left(R, J_{R}(z)\right)=\coprod_{j \in \mathbb{N}} \mathfrak{Q}_{j}(R, z)=$ the ideal tree of $z$ in $R$, and for any nonzero element $F$ in $R$ we define: 
$\mathfrak{P}_{j}(R, F)=\mathfrak{P}_{j}(R, F R)=j$-th layer of the singularity tree of $F$ in $R$, $\mathfrak{P}(R, F)=\mathfrak{P}(R, F R)=\coprod_{j \in \mathbb{N}} \mathfrak{P}_{j}(R, F)=$ the singularity tree of $F$ in $R$.

(2A) Transforms of elements. Given any $T \in Q(R)$ and any nonzero ideal $I$ in $R$, we shall now extend the idea of the transform of $I$ to the idea of the transform of specific elements of $I$. Given any generators $x, y$ of $M(R)$ and any coefficient set $\kappa$ of $R$, as explained in Section 2 of [Ab11, there is a unique finite QDT sequence $\left(R_{j}, x_{j}, y_{j}, \kappa_{j}\right)_{0 \leq j \leq \nu}$ of $(R, x, y, \kappa)$ with $T=R_{\nu}$. We put

$$
(R, T)(x, y, \kappa)=\left(x_{\nu}, y_{\nu}, \kappa_{\nu}\right)
$$

and we call $\left(x_{\nu}, y_{\nu}, \kappa_{\nu}\right)$ the $(R, T)$-transform of $(x, y, \kappa)$. For $0 \leq j \leq \nu$ let $I_{j}=\left(R, R_{j}\right)(I)$ and $d_{j}=\operatorname{ord}_{R_{j}} I_{j}$ and $z_{j}=\left\{\begin{array}{l}y_{j} \text { if } j \neq 0 \text { and } x_{j-1} / y_{j-1} \in M\left(R_{j}\right) \\ x_{j} \text { if } j \neq 0 \text { and } x_{j-1} / y_{j-1} \notin M\left(R_{j}\right) \\ x_{j} \text { if } j=0 .\end{array}\right.$

Note that then

$$
I_{0}=I \text { and } I_{j-1} R_{j}=I_{j}\left(z_{j}^{d_{j}} R_{j}\right) \text { for } 1 \leq j \leq \nu .
$$

Given any finite number of nonzero elements $f^{(1)}, \ldots, f^{(p)}$ in $I$, clearly there exist unique nonzero elements $f_{j}^{(1)}, \ldots, f_{j}^{(p)}$ in $I_{j}$ for $0 \leq j \leq \nu$ such that $f_{0}^{(1)}=f^{(1)}, \ldots, f_{0}^{(p)}=f^{(p)}$ and $f_{j-1}^{(1)}=f_{j}^{(1)} z_{j}^{d_{j}}, \ldots, f_{j-1}^{(p)}=f_{j}^{(p)} z_{j}^{d_{j}}$ for $1 \leq j \leq \nu$. We put

$$
(R, x, y, \kappa, T, I)\left(f^{(1)}, \ldots, f^{(p)}\right)=\left(f_{\nu}^{(1)}, \ldots, f_{\nu}^{(p)}\right)
$$

and we call $\left(f_{\nu}^{(1)}, \ldots, f_{\nu}^{(p)}\right)$ the $(R, x, y, \kappa, T, I)$-transform of $\left(f^{(1)}, \ldots, f^{(p)}\right)$. Note that for $0 \leq j \leq \nu$ we clearly have

$$
\left\{\begin{array}{l}
\left(R, R_{j}\right)(x, y, \kappa)=\left(x_{j}, y_{j}, \kappa_{j}\right) \text { with }\left(R_{j}, T\right)\left(x_{j}, y_{j}, \kappa_{j}\right)=\left(x_{\nu}, y_{\nu}, \kappa_{\nu}\right), \\
\text { and }\left(R, R_{j}\right)(I)=I_{j} \text { with }\left(R_{j}, T\right)\left(I_{j}\right)=I_{\nu}, \\
\text { and }\left(R, x, y, \kappa, R_{j}, I\right)\left(f^{(1)}, \ldots, f^{(p)}\right)=\left(f_{j}^{(1)}, \ldots, f_{j}^{(p)}\right) \\
\text { with }\left(R_{j}, x_{j}, y_{j}, \kappa_{j}, T, I_{j}\right)\left(f_{j}^{(1)}, \ldots, f_{j}^{(p)}\right)=\left(f_{\nu}^{(1)}, \ldots, f_{\nu}^{(p)}\right) \\
\text { moreover, if } I=\left(f^{(1)}, \ldots, f^{(p)}\right) R, \text { then } I_{j}=\left(f_{j}^{(1)}, \ldots, f_{j}^{(p)}\right) R_{j} .
\end{array}\right.
$$

(2B) Tangent directions. Given any generators $x, y$ of $M(R)$ and any coefficient set $\kappa$ of $R$, picturing $R$ as the local ring of a simple point on a surface, we can describe the tangent directions at that point thus. Let $K=H(R)=R / M(R)$, and let $X, Y$ be indeterminates. Given any $0 \neq F \in M(R)$ we can write

$$
F=\sum_{i+j=d} \widetilde{F}_{i j} x^{i} y^{j} \quad \text { with } \quad d=\operatorname{ord}_{R} F \quad \text { and } \quad \widetilde{F}_{i j} \in R .
$$

We can think of $F=0$ as the local equation of a curve on the surface at that point. As in Remark (4.0) of [Ab11], we define the initial form

$$
\operatorname{info}(F)=\operatorname{info}_{(R, x, y)} F
$$

of $F$ (relative to $R, x, y$ ) to be the nonzero homogeneous polynomial

$$
\bar{F}=\bar{F}(X, Y) \in K[X, Y]=\operatorname{grad}(R)
$$


of degree $d$ with coefficients in $K$ obtained by putting

$$
\bar{F}(X, Y)=\sum_{i+j=d} H_{R}\left(\widetilde{F}_{i j}\right) X^{i} Y^{j} .
$$

From that remark, recall the definitions of $\operatorname{deg}_{X} f, \operatorname{deg}_{Y} f, \operatorname{deg}_{X, Y} f, \operatorname{ord}_{X} f, \operatorname{ord}_{Y} f$, $\operatorname{ord}_{X, Y} f$, info ${ }_{X, Y} f$, with $f=f(X, Y) \in S[X, Y]$, where $S$ is any ring. For any subset $\bar{S}$ of $S$ such that 0 and 1 belong to $S$, by $\bar{S}[X, Y]$ we denote the set of all $f=f(X, Y) \in S[X, Y]$ whose coefficients belong to $\bar{S}$. By $\bar{S}[X, Y]^{h}$ we denote the set of all $\lambda=\lambda(X, Y) \in \bar{S}[X, Y]$ which are homogeneous of some degree $\omega \in \mathbb{N}$, i.e.,

$$
\lambda(X, Y)=\sum_{0 \leq i \leq \omega} \lambda_{i} X^{i} Y^{\omega-i} \text { with } \lambda_{i} \in \bar{S} .
$$

By $\bar{S}[X, Y]^{h m}$ we denote the set of all $\lambda \in \bar{S}[X, Y]^{h}$ which are $Y$-monic; i.e., for a (unique) nonnegative integer $\iota \leq \omega$ we have $\lambda_{\iota}=1$ and $\lambda_{i}=0$ for all $0 \leq i<\iota$. Note that $\iota=\operatorname{ord}_{X} \lambda$. In the case of $\bar{S}=\kappa \subset R=S$, by $\kappa[X, Y]^{h m i}$ we denote the set of all $\lambda=\lambda(X, Y) \in \kappa[X, Y]^{h m}$ such that

$$
H_{R}(\lambda)=H_{R}(\lambda)(X, Y)=\sum_{0 \leq i \leq \omega} H_{R}\left(\lambda_{i}\right) X^{i} Y^{\omega-i}
$$

is irreducible in $K[X, Y]$. Note that this is so iff either $\iota=\omega=1$ with $\lambda(X, Y)=X$ or $\iota=0<\omega \in \mathbb{N}_{+}$and

$$
\lambda(1, Z)=Z^{\omega}+\sum_{1 \leq i \leq \omega} H_{R}\left(\lambda_{i}\right) Z^{\omega-i}
$$

is irreducible in the univariate polynomial ring $K[Z]$. Note that

$$
\lambda(X, Y) \mapsto \mu(Z)=\lambda(1, Z) \text { gives a bijection } \kappa[X, Y]^{h m i} \backslash\{X\} \rightarrow \kappa[Z]^{m i},
$$

where $\kappa[Z]^{m i}$ is the set of all $\mu(Z)$ described in Section 2 of Ab11. The material around that description yields the bijection

$$
\delta: \kappa[X, Y]^{h m i} \rightarrow Q_{1}(R), \text { where } \delta(\lambda)=R^{\prime} \in Q_{1}(R) \text { with } M\left(R^{\prime}\right)=\left(x^{\prime}, y^{\prime}\right) R^{\prime}
$$

given by

$$
\left(x^{\prime}, y^{\prime}\right)= \begin{cases}(\lambda(x / y, 1), y) & \text { if } y / x \notin R^{\prime}=\delta(\lambda), \text { i.e., equivalently if } \lambda=X \\ (x, \lambda(1, y / x)) & \text { if } y / x \in R^{\prime}=\delta(\lambda), \text { i.e., equivalently if } \lambda \neq X\end{cases}
$$

Let $\kappa^{\prime}$ be the coefficient set of $R^{\prime}$ described in the said material. We call $\left(R^{\prime}, x^{\prime}\right.$, $\left.y^{\prime}, \kappa^{\prime}\right)$ the QDT of $(R, x, y, \kappa)$ in the tangent direction $\lambda$. We call $\delta$ the tangential bijection.

For any $\lambda \in \kappa[X, Y]^{h m i}$ let $R^{\prime}=\delta(\lambda)$. Then obviously

$$
\left\{\begin{array}{l}
\text { upon letting } \omega(\lambda)=\operatorname{deg}_{X, Y} H_{R}(\lambda), \\
\text { we have } \omega(\lambda)=\left[R^{\prime} / M\left(R^{\prime}\right): K\right] \\
\text { where we regard } R^{\prime} / M\left(R^{\prime}\right) \text { to be an overfield of } K
\end{array}\right.
$$

Let

$$
(z, \zeta)= \begin{cases}(y, x / y) & \text { if } \lambda=X \\ (x, y / x) & \text { if } \lambda \neq X\end{cases}
$$


Then clearly

$$
\left\{\begin{array}{l}
z \in M\left(R^{\prime}\right) \backslash M\left(R^{\prime}\right)^{2} \text { with } M(R) R^{\prime}=z R^{\prime} \\
\text { and upon letting } F^{\prime}=F / z^{d} \\
\text { we have }\left(R, x, y, \kappa, R^{\prime}, F R\right)(F)=F^{\prime} \in R^{\prime} \backslash z R^{\prime}, \\
\text { and upon letting } d^{\prime}=\operatorname{ord}_{R^{\prime}} F^{\prime} \text { with } e^{\prime}=\operatorname{ord}_{V^{\prime}} \alpha\left(F^{\prime}\right), \\
\text { where } \alpha: R^{\prime} \rightarrow V^{\prime}=R^{\prime} / z R^{\prime} \text { is the residue class epimorphism, } \\
\text { we have that } d^{\prime}, e^{\prime} \text { are nonnegative integers with } d^{\prime} \leq e^{\prime} \\
\text { such that: } d^{\prime}=0 \Leftrightarrow e^{\prime}=0 . \\
\text { Note that } e^{\prime}=\operatorname{ord}_{\left(R^{\prime} / z R^{\prime}\right)} F^{\prime} \text { according to Remark (4.0) of Ab11]. }
\end{array}\right.
$$

Next let us prove the following:

$$
\left\{\begin{array}{l}
\text { Let } e \text { be the unique nonnegative integer such that } \\
\text { (4) } \bar{F}(X, Y) \in\left(H_{R}(\lambda)^{e} K[X, Y]\right) \backslash\left(H_{R}(\lambda)^{e+1} K[X, Y]\right) . \\
\text { Then } e^{\prime}=e .
\end{array}\right.
$$

By symmetry the case of $\lambda=X$ follows from the case of $\lambda=Y$, and hence in proving (2.4) we may assume that $\lambda \neq X$. Now $(z, \zeta)=(x, y / x)$ and, in view of the references to $\mathrm{Ab3}$ and $\mathrm{Ab} 8$ given at the beginning of this Section 2, there is a unique epimorphism $\theta: A=R[\zeta] \rightarrow K[Z]$ with $\operatorname{ker}(\theta)=M(R) A=z A$ such that $\theta(\zeta)=Z$ and $\theta(r)=H_{R}(r)$ for all $r \in R$. Equations (1) to (4) tell us that

$$
F^{\prime} \in A \text { with } \theta\left(F^{\prime}\right) \in\left(\mu(Z)^{e} K[Z]\right) \backslash\left(\mu(Z)^{e+1} K[Z]\right) .
$$

Let $\beta: A \rightarrow R^{\prime}=A_{(z, \mu(\zeta)) A}$ and let $\gamma: K[Z] \rightarrow V=K[Z]_{\mu(Z) K[Z]}$ be the localization injections. Then by $(2.3)$ and (5) we have

$$
\operatorname{ord}_{V^{\prime}} \alpha\left(\beta\left(F^{\prime}\right)\right)=e^{\prime} \text { and } \operatorname{ord}_{V} \gamma\left(\theta\left(F^{\prime}\right)\right)=e .
$$

Now the permutability of localization and surjection given in (T11) on page 139 of Ab8 yields a unique isomorphism $\gamma^{\prime}: V \rightarrow V^{\prime}$ such that $\gamma^{\prime} \gamma \theta=\alpha \beta$, i.e., such that the diagram

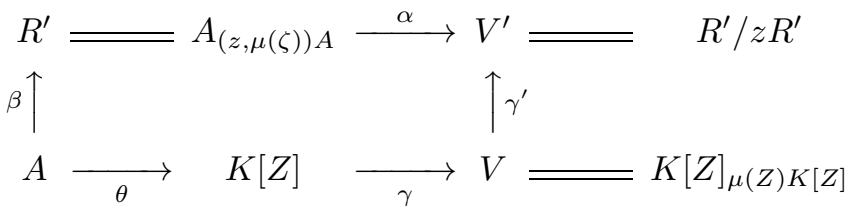

commutes. Therefore by (6) we get $e^{\prime}=e$.

(2C) Tangents of curves. To get the tangents to the curve $C$ whose equation is $F=0$ at the point $P$ whose local ring is $R$, by factoring $\bar{F}$ we obtain

$$
\bar{F}(X, Y)=\widehat{F} \prod_{1 \leq i \leq h} H_{R}\left(\bar{F}_{i}\right)(X, Y)^{e_{i}}
$$

with

$$
\widehat{F} \in K^{\times} \text {and } \operatorname{deg}_{X, Y} H_{R}\left(\bar{F}_{i}\right)=\omega_{i} \in \mathbb{N}_{+},
$$

where $h, e_{1}, \ldots, e_{h}$ are positive integers and $\bar{F}_{1}, \ldots, \bar{F}_{h}$ are pairwise distinct members of $\kappa[X, Y]^{h m i}$. These members may be viewed as the required tangents, 
the positive integers $e_{1}, \ldots, e_{h}$ may be called the corresponding tangential multiplicities, and the positive integers $\omega_{1}, \ldots, \omega_{h}$ may be called the corresponding tangential degrees. Clearly

$$
e_{1} \omega_{1}+\cdots+e_{h} \omega_{h}=d
$$

For $1 \leq i \leq h$ we let

$$
z_{i}= \begin{cases}y & \text { if } \bar{F}_{i}=X \\ x & \text { if } \bar{F}_{i} \neq X\end{cases}
$$

with

$$
F_{i}^{\prime}=F / z_{i}^{d} \text { and } R_{i}^{\prime}=\delta\left(\bar{F}_{i}\right)
$$

and upon letting

$$
\alpha_{i}: R_{i}^{\prime} \rightarrow V_{i}^{\prime}=R_{i}^{\prime} / z_{i} R_{i}^{\prime}
$$

be the residue class epimorphism and observing that

$$
F_{i}^{\prime} \in R_{i}^{\prime}=\text { a two-dimensional regular local domain }
$$

with

$$
V_{i}^{\prime}=\text { a one-dimensional regular local domain }
$$

we let

$$
d_{i}^{\prime}=\operatorname{ord}_{R_{i}^{\prime}} F_{i}^{\prime} \text { and } e_{i}^{\prime}=\operatorname{ord}_{V_{i}^{\prime}} \alpha_{i}\left(F_{i}^{\prime}\right) .
$$

Recalling that $\mathfrak{P}_{1}(R, F)=\left\{T \in Q_{1}(R):(R, T)(F R) \neq T\right\}$, by (2.2) to (2.4) we get the following:

$$
\left\{\begin{array}{l}
R_{1}^{\prime}, \ldots, R_{h}^{\prime} \text { are exactly all the distinct members of } \mathfrak{P}_{1}(R, F) \\
\text { and for } 1 \leq i \leq h \text { we have that }\left(R, x, y, \kappa, R_{i}^{\prime}, F R\right)(F)=F_{i}^{\prime} \\
\text { and } M(R) R_{i}^{\prime}=z_{i} R_{i}^{\prime} \text { with }\left[R_{i}^{\prime} / M\left(R_{i}^{\prime}\right): K\right]=\omega_{i} \\
\text { and } d_{i}^{\prime}, e_{i}^{\prime} \text { are positive integers with } d_{i}^{\prime} \leq e_{i}^{\prime}=e_{i} .
\end{array}\right.
$$

By (2.5) and (2.6) we see that

$$
\text { for } 1 \leq i \leq h \text { we have } d_{i}^{\prime} \leq d
$$

and

$$
\left\{\begin{array}{l}
\text { if } e_{i}=1 \text { for some } i \in\{1, \ldots, h\} \\
\text { then for that } i \text { we have } M\left(R_{i}^{\prime}\right)=\left(F_{i}^{\prime}, z_{i}\right) R_{i}^{\prime}
\end{array}\right.
$$

and

$$
\left\{\begin{array}{l}
\text { if } d_{i}^{\prime} \geq d \text { for some } i \in\{1, \ldots, h\}, \\
\text { then } h=1=\omega_{1}=\left[R_{1}^{\prime} / M\left(R_{1}^{\prime}\right): K\right]=1 \\
\text { with } d=d_{1}^{\prime}=e_{1}^{\prime}=e_{1} .
\end{array}\right.
$$

(2D) Geometric language. Referring to pages 131-143 of my engineering book Ab6], assertion (2.6) to (2.9) can be paraphrased geometrically thus. The total transform of $C$, obtained by applying a QDT centered at $P$, consists of the exceptional line $E$ together with the (proper) transform $C^{\prime}$ of $C$. The exceptional line $E$ cuts the proper transform $C^{\prime}$ at points $P_{1}, \ldots, P_{h}$ with intersection multiplicities $e_{1}^{\prime}=e_{1}, \ldots, e_{h}^{\prime}=e_{h}$. Letting $d_{1}^{\prime}, \ldots, d_{h}^{\prime}$ be the multiplicities of $C^{\prime}$ at $P_{1}, \ldots, P_{h}$, we have $d_{1}^{\prime} \leq e_{1}^{\prime}, \ldots, d_{h}^{\prime} \leq e_{h}^{\prime}$ with $e_{1}^{\prime} \omega_{1}+\cdots+e_{h}^{\prime} \omega_{h}=d$. After suitable relabelling, $R_{1}^{\prime}, \ldots, R_{h}^{\prime}$ may be regarded as the local rings of $P_{1}, \ldots, P_{h}$, and then $z_{1}=0, \ldots, z_{h}=0$ become the equations of $E$ at $P_{1}, \ldots, P_{h}$, whereas 
$F_{1}^{\prime}=0, \ldots, F_{h}^{\prime}=0$ become the equations $C^{\prime}$ at $P_{1}, \ldots, P_{h}$, respectively. Assertion (2.9) corresponds to the second paragraph on page 135 of Ab6 saying that: "Thus, if after one QDT there is no drop in multiplicity, then ... and the line $X=0$ is not tangent to $C^{\prime}$ at $P_{1} . "$

(2E) Magnitude. The above cited pages 131-143 constitute Lecture 18 of the book Ab6]. The rest of that Lecture 18 is a concrete version my Arcata paper Ab5, which itself was streamlined in my PAMS paper Ab7. The main idea is to introduce the concept of the magnitude, abbreviated as mag, of the nonzero principal ideal $J=F R$ in $R$. We allow $J$ to be the unit ideal, i.e., $d$ to be zero. The magnitude will be a nonnegative rational number or $\infty$; its integral part will tell us how many QDTs will be needed to decrease the order $d$ of $J$. Here is a concise summary of the matter.

Expanding in the completion $\widehat{R}$ of $R$ we write

$$
F=\sum_{(i, j) \in \mathbb{N} \times \mathbb{N}} F_{i j} x^{i} y^{j} \text { with } F_{i j} \in \kappa .
$$

For any $q \in \mathbb{Q}_{+}=$the set of all positive rationals, we define the weighted order and the weighted initial form by putting

$$
\operatorname{word}_{(R, x, y, 1, q)} F=W,
$$

where

$$
W=\min \left\{i+q j: F_{i j} \neq 0\right\} \in \mathbb{Q}_{+}
$$

and

$$
\operatorname{winfo}_{(R, x, y, 1, q)} F=\sum_{(i, j) \in U} H_{R}\left(F_{i j}\right) X^{i} Y^{j} \in K[X, Y]
$$

where

$$
U=\{(i, j) \in \mathbb{N} \times \mathbb{N}: i+j q=W\}
$$

Upon letting

$$
D=\operatorname{ord}_{R / x R} J \in \mathbb{N} \cup\{\infty\}
$$

by Section 7 of [Ab5] we see that if $D \neq \infty$ and $J \neq y^{d} R$, then for a unique $q \in \mathbb{Q}_{+}$ we have $(0, D) \in U \neq\{(0, D)\}$. We let

$$
\epsilon= \begin{cases}q & \text { if } D \neq \infty \text { and } J \neq y^{d} R \\ \infty & \text { if } D \neq \infty \text { and } J=y^{d} R \\ 0 & \text { if } D=\infty\end{cases}
$$

and we define $\operatorname{mag}(R, J, x, y)$ by putting

$$
\operatorname{mag}(R, J, x, y)=\epsilon .
$$

If $D \neq \infty$ with $J \neq y^{d} R$, then we define the normalized initial form by putting

$$
\operatorname{ninfo}_{(R, x, y)} J=H_{R}\left(F_{0 D}\right)^{-1} \operatorname{winfo}_{(R, x, y, 1, \epsilon)} F \text {. }
$$

We say that $\operatorname{ninfo}_{(R, x, y)} J$ is a monic power to mean that

$$
D \neq \infty \text { with } J \neq y^{d} R \text { and } \operatorname{ninfo}_{(R, x, y)} J=(Y-p(X))^{D} \text { with } p(X) \in K[X]^{\times} .
$$

Note that mag and ninfo are independent of the quantities $\kappa$ and $F$, and hence these quantities are not included in the notation. Likewise, winfo is independent of $\kappa$ and hence $\kappa$ is not included in the notation. Using (2.6) and Section 7 of Ab5] we can show that: 
Proposition (2.10). In the above notation we have the following.

(2.10.1) $\epsilon \in\{0\} \cup \mathbb{Q}_{+} \cup\{\infty\}$.

(2.10.2) Assume that $D \neq \infty$ with $J \neq y^{d} R$. Then $D \in \mathbb{N}_{+}$with $d \in \mathbb{N}_{+}$and $\epsilon \in \mathbb{Q}_{+}$with $D ! \epsilon \in \mathbb{N}_{+}$. Moreover, $\epsilon<1 \Leftrightarrow D \neq d \Leftrightarrow D>d$.

(2.10.3) Assume that $\operatorname{ninfo}_{(R, x, y)} J$ is a monic power. Then $D=d \in \mathbb{N}_{+}$with $\epsilon \in \mathbb{N}_{+}$and $\operatorname{ninfo}_{(R, x, y)} J=\left(Y-H_{R}(c)\right)^{d}$ for a unique nonzero $c \in \kappa$. Moreover, $\epsilon$ increases by changing $y$ to $y-c x$, i.e., $(x, y-c x) R=M(R)$ with $\operatorname{mag}(R, J, x, y-$ $c x)>\epsilon$.

(2.10.4) Assume that $\epsilon>1$. Then $\mathfrak{P}_{1}(R, J)$ contains exactly one member $R^{\prime}$, and upon letting $\left(R^{\prime}, x^{\prime}, y^{\prime}, \kappa^{\prime}\right)$ be the corresponding $Q D T$ of $(R, x, y, \kappa)$ we have $\left(x^{\prime}, y^{\prime}, \kappa^{\prime}\right)=(x, y / x, \kappa)$, and upon letting $J^{\prime}=\left(R, R^{\prime}\right)(J)$ with $\epsilon^{\prime}=\operatorname{mag}\left(R^{\prime}, J^{\prime}, x^{\prime}, y^{\prime}\right)$ we have $\epsilon^{\prime}=\epsilon-1$ with the convention that $\infty \pm \theta=\infty$ for all $\theta \in \mathbb{Q}$, and upon letting

$$
F^{\prime}=\left(R, x, y, \kappa, R^{\prime}\right)(F) \text { with } d^{\prime}=\operatorname{ord}_{R^{\prime}} J^{\prime} \text { and } D^{\prime}=\operatorname{ord}_{R^{\prime} / x^{\prime} R^{\prime}} J^{\prime}
$$

we have $F^{\prime}=F / x^{d}$ with $D^{\prime}=d \geq d^{\prime}$, and if $\epsilon \neq \infty$, then upon letting

$$
\Omega(X, Y)=\operatorname{winfo}_{(R, x, y, 1, \epsilon)} F \in K[X, Y]
$$

and

with

$$
\Omega^{\prime}(X, Y)=\operatorname{winfo}_{\left(R^{\prime}, x^{\prime}, y^{\prime}, 1, \epsilon^{\prime}\right)} F^{\prime} \in K[X, Y]
$$

$$
\Lambda(X, Y)=\operatorname{ninfo}_{(R, x, y)} J \in K[X, Y] \text { and } \Lambda^{\prime}(X, Y)=\operatorname{ninfo}_{\left(R^{\prime}, x^{\prime}, y^{\prime}\right)} J^{\prime} \in K[X, Y]
$$

we have

$$
\Omega(X, X Y)=X^{d} \Omega^{\prime}(X, Y) \text { with } \Lambda(X, X Y)=X^{d} \Lambda^{\prime}(X, Y)
$$

and

$$
\operatorname{ninfo}_{(R, x, y)} J \text { is a monic power } \Leftrightarrow \operatorname{ninfo}_{\left(R^{\prime}, x^{\prime}, y^{\prime}\right)} J^{\prime} \text { is a monic power. }
$$

Inspired by (2.9) and the definition of $\epsilon$, we make the following definitions. We say that $(R, J)$ is resolved to mean that $J=y^{* d} R$ for some $y^{*} \in M(R) \backslash M(R)^{2}$. We say that $(R, J)$ is unitangent to mean that $J=\left(y^{* d}+\widetilde{y}\right) R$ for some $y^{*} \in M(R) \backslash$ $M(R)^{2}$ and $\widetilde{y} \in M(R)^{d+1}$. Recall that a ring $A$ is said to be pseudogeometric if $A$ is Noetherian and, for any prime ideal $P$ in $A$, the integral closure of $A / P$ in any finite algebraic field extension of the quotient field of $A / P$ is a finite $(A / P)$-module.

We let

$$
B(R, J)=\left\{\left(x^{*}, y^{*}\right):\left(x^{*}, y^{*}\right) R=M(R) \text { and } \operatorname{ord}_{R / x^{*}}=\operatorname{ord}_{R} J\right\}
$$

and

$$
\widehat{e}= \begin{cases}\max \left\{\operatorname{mag}\left(R, J, x^{*}, y^{*}\right):\left(x^{*}, y^{*}\right) \in B(R, J)\right\} & \text { if } B(R, J) \neq \emptyset \\ 1 & \text { if } B(R, J)=\emptyset\end{cases}
$$

where max denotes the least upper bound, and we define mag $[R](J)$ by putting

$$
\operatorname{mag}[R](J)=\widehat{\epsilon} .
$$

We define the integral magnitude inmag $[R](J)$ to be the maximum $s \in \mathbb{N}_{+} \cup\{\infty\}$ for which there exists a sequence $\left(R_{j}, J_{j}\right)_{0 \leq j<s}$ with $\left(R_{0}, J_{0}\right)=(R, J)$ such that for $1 \leq u<s$ we have $R_{j} \in Q_{1}\left(R_{j-1}\right)$ with $J_{j}=\left(R_{j-1}, R_{j}\right)\left(J_{j-1}\right)$ and $\operatorname{ord}_{R_{j}} J_{j}=d$. By the integral part $[q]$ of any $q \in \mathbb{Q}$ we mean the largest integer $\leq q$; we also put $[\infty]=\infty$. 
Using (2.10) and Sections 2 to 9 of [Ab5] we can show that:

Proposition (2.11). In the above notation with $\operatorname{inmag}[R](J)=s$ we have the following.

(2.11.1) $1 \leq \widehat{\epsilon} \in \mathbb{Q}_{+} \cup\{\infty\}$.

(2.11.2) $d=0 \Rightarrow(R, J)$ is resolved $\Rightarrow s=\widehat{\epsilon}=\infty$.

(2.11.3) $\widehat{\epsilon}>1 \Leftrightarrow \widehat{\epsilon} \neq 1 \Leftrightarrow(R, J)$ is unitangent.

(2.11.4) $\widehat{\epsilon} \neq \infty \Rightarrow d ! \widehat{\epsilon} \in \mathbb{N}_{+}$.

$(2.11 .5) \widehat{\epsilon}<2 \Rightarrow \operatorname{ord}_{R^{\prime}}\left(R, R^{\prime}\right)(J)<d$ for all $R^{\prime} \in Q_{1}(R)$.

(2.11.6) Assume that $\widehat{\epsilon} \geq 2$. Then $\mathfrak{P}_{1}(R, J)$ contains exactly one member $R^{\prime}$, and upon letting $J^{\prime}=\left(R, R^{\prime}\right)(J)$ we have $B\left(R^{\prime}, J^{\prime}\right) \neq \emptyset$ and $\operatorname{mag}\left[R^{\prime}\right]\left(J^{\prime}\right)=\widehat{\epsilon}-1$ with the convention that $\infty \pm \theta=\infty$ for all $\theta \in \mathbb{Q}$.

(2.11.7) $\epsilon \geq \widehat{\epsilon} \neq \infty \Rightarrow \epsilon=\widehat{\epsilon}$ and $\operatorname{ninfo}_{(R, x, y)} J$ is not a monic power.

(2.11.8) $1 \leq \epsilon<\widehat{\epsilon} \Rightarrow \operatorname{ninfo}_{(R, x, y)} J$ is a monic power.

(2.11.9) $1<\widehat{\epsilon} \neq \infty \Rightarrow \operatorname{mag}\left(R, J, x^{*}, y^{*}\right)=\widehat{\epsilon}$ for some $\left(x^{*}, y^{*}\right) \in B(R, J)$.

(2.11.10) $\widehat{\epsilon}=\infty \Rightarrow(\widehat{R}, J \widehat{R})$ is resolved.

(2.11.11) $R$ is pseudogeometric and $\widehat{\epsilon}=\infty \Rightarrow(R, J)$ is resolved.

(2.11.12) $d \neq 0 \Rightarrow s=[\widehat{\epsilon}$. Also see (9.4) and (9.14) of [Ab5].

\section{Bushes AND THICKETS}

Let $R$ be a two-dimensional regular local domain. We shall now introduce several types of subsets of $Q(R)$.

Let $B \subset Q(R)$. We define the breadth of $B$ by putting

$$
\beta(B)=\max \left\{\left|Q_{j}(R) \cap B\right|: j \in \mathbb{N}\right\}
$$

and we note that $\beta(B) \in \mathbb{N} \cup\{\infty\}$ and $\beta(B)=0 \Leftrightarrow B=\emptyset$. By a pine of $B$ we mean an infinite QDT sequence $S=\left(S_{j}\right)_{0 \leq j<\infty}$ of some member $S_{0}$ of $B$ such that for all $j \in \mathbb{N}$ we have $Q_{j}\left(S_{1}\right) \cap B=\left\{S_{j+1}\right\}$; we call $S_{0}, S_{1}, S_{j}$ the base, subbase, $j$-th component of $S$ respectively. By a subpine of the pine $S$ we mean a pine $S^{\prime}=\left(S_{j}^{\prime}\right)_{0 \leq j<\infty}$ of $B$ such that for some $j^{\prime} \in \mathbb{N}$ we have $S_{j}^{\prime}=S_{j^{\prime}+j}$ for all $j \in \mathbb{N}$; if $j^{\prime} \neq 0$, then we call $S^{\prime}$ a proper subpine of $S$. By a maximal pine of $B$ we mean a pine of $B$ which is not a proper subpine of any pine of $B$. All these definitions are relative to $R$.

Let $B \subset Q(R)$ and let $I$ be a nonzero ideal in $R$. A pine $S=\left(S_{j}\right)_{0 \leq j<\infty}$ of $B$ is said to be stationary at $(R, I)$ if there exist (unique) nonnegative integers

$$
r=\operatorname{rlen}(R, S, I) \text { and } u=\operatorname{uord}(R, S, I),
$$

which we respectively call the reduced length and ultimate order of $(R, S, I)$, such that

$$
\left\{\begin{array}{l}
\text { for all } r \leq j \in \mathbb{N} \text { we have } \\
{\left[S_{j} / M\left(S_{j}\right): S_{r} / M\left(S_{r}\right)\right]=1 \text { and } \operatorname{ord}_{S_{j}}\left(R, S_{j}\right)(I)=u}
\end{array}\right.
$$

and

$$
\left\{\begin{array}{l}
\text { for all } r>j \in \mathbb{N} \text { we have } \\
\text { either }\left[S_{r} / M\left(S_{r}\right): S_{j} / M\left(S_{j}\right)\right]>1 \text { or } \operatorname{ord}_{S_{j}}\left(R, S_{j}\right)(I)>u
\end{array}\right.
$$

When the above conditions are satisfied, we define the solidified subpine $S^{\prime}=$ $\left(S_{j}^{\prime}\right)_{0 \leq j<\infty}$ of $S$ by putting $S_{j}^{\prime}=S_{j+r}$ for all $j \in \mathbb{N}$. $B$ is said to be stable at $(R, I)$ if every pine of $B$ is stationary at $(R, I)$. $B$ is said to be strongly square free at $(R, I)$ if for every pine $S$ of $B$ which is stationary at $(R, I)$ we have uord $(R, S, I)=1$. 
Finally, we say that $(R, I)$ is strongly square free if $\mathfrak{P}(R, I)$ is strongly square free at $(R, I)$.

By a bush at $R$ we mean a union of finite QDT sequences of $R$. By a thicket at $R$ we mean a union of infinite QDT sequences of $R$. Equivalently, a thicket at $R$ is a bush $B$ at $R$ such that for every $T \in B$ we have $Q_{1}(T) \cap B \neq \emptyset$. Formally speaking, a bush is a subset $B$ of $Q(R)$ such that $B=\left\{R_{i j}: i \in \Omega\right.$ and $\left.0 \leq j \leq \nu_{i}\right\}$ for some set $\Omega$ of finite QDT sequences $\left(R_{i j}\right)_{0 \leq j \leq \nu_{i}}$ of $R$ with $i \in \Omega$. Likewise, a thicket is a subset $B$ of $Q(R)$ such that $B=\left\{R_{i j}: i \in \Omega\right.$ and $\left.0 \leq j<\infty\right\}$ for some set $\Omega$ of infinite QDT sequences $\left(R_{i j}\right)_{0 \leq j<\infty}$ of $R$ with $i \in \Omega$; now upon letting $R_{i l j}=R_{i j}$ we get a finite QDT sequence $\left(R_{i l j}\right)_{0 \leq j \leq l}$ of $R$ for all $(i, l) \in \Omega \times \mathbb{N}$ with $B=\left\{R_{i l j}: i \in \Omega, l \in \mathbb{N}, 0 \leq j \leq l\right\}$ showing that every thicket is a bush. Note that a thicket of finite nonzero breadth $\beta$ has exactly $\beta$ maximal pines.

In Figure 18.2 on page 132 of $\mathrm{Ab} 6$, the finite tree emanating from $P$ is a bush but not a thicket. Figure 5 on page 426 of $\mathrm{Ab} 4$ with the dotted branches going to infinity is a thicket and hence also a bush; the dotted branches extended downwards give the maximal pines.

By a stable thicket at $(R, I)$ we mean a thicket $B$ at $R$ such that $B$ is stable at $(R, I)$. By $(2.5)$ and (2.9) together with Section 2 of [Ab11] we get:

Theorem (3.1). For any $0 \neq F \in R, \mathfrak{P}(R, F R)$ is a stable thicket at $(R, F R)$ and its breadth is finite. For any $M(R)$-primary ideal $J$ in $R, \mathfrak{Q}(R, J)$ is a finite bush at $R$.

Note (3.2). A direct characterization of a thicket $B$ of finite nonzero breadth can be given by saying that $B$ is subset of $Q(R)$ such that: (i) $R \in B$, (ii) for every $T \in B$ we have $Q_{1}(T) \cap B \neq \emptyset$, (iii) for every finite QDT sequence $\left(R_{j}\right)_{0 \leq j \leq \nu}$ of $R$ with $R_{\nu} \in B$ we have $R_{j} \in B$ for $0 \leq j<\nu$, and (iv) there exists a (unique) positive integer $\beta=\beta(B)$, called the breadth of $B$, together with a (unique) nonnegative integer $\mu=\mu(B)$, called the height of $B$, for which $\mid\left(Q_{0}(R) \cap B|\leq|\left(Q_{1}(R) \cap B \mid \leq\right.\right.$ $\cdots \leq \mid\left(Q_{\mu-1}(R) \cap B \mid<\beta\right.$ and $\left|Q_{\mu} \cap B\right|=\left|Q_{\mu+1}(R) \cap B\right|=\cdots=\beta$.

\section{QDTS AND GENERIC QDTS}

As in the beginning of Section 2, let $L^{t}=L(t)$, where $t$ is an indeterminate over a field $L$, and let $\rho^{t}: \rho(L) \rightarrow \rho\left(L^{t}\right)$ be the map given by $S \mapsto S^{t}$, where $\rho(L)$ and $\rho\left(L^{t}\right)$ are the sets of all subrings of $L$ and $L^{t}$ respectively. In view of (T30) on page 233 of $\mathrm{Ab8}$, we see that:

$\left\{\begin{array}{l}\text { If } S \in \rho(L) \text { is a local domain, then } S^{t} \text { is a local domain with } \\ \operatorname{dim}\left(S^{t}\right)=\operatorname{dim}(S) \text { and } M\left(S^{t}\right)=M(S) S^{t} \text { and } S^{t} \cap L=S . \\ \text { If } S \in \rho(L) \text { is a regular local domain, then } S^{t} \text { is a regular local domain. }\end{array}\right.$

Now let $R$ be a two-dimensional regular local domain with quotient field $L$, and let $F, G$ be nonzero elements in $M(R)$ which generate an $M(R)$-primary ideal $J$ in $R$. Taking over the setup of Subsections (2B) and (2C), and extending it to $G$, we can write

$$
G=\sum_{i+j=d(G)} \widetilde{G}_{i j} x^{i} y^{j} \quad \text { with } \quad d(G)=\operatorname{ord}_{R} G \quad \text { and } \quad \widetilde{G}_{i j} \in R
$$


and

$(2 \mathrm{G})$

$$
\bar{G}=\bar{G}(X, Y)=\operatorname{info}(G)=\operatorname{info}_{(R, x, y)} G=\sum_{i+j=d(G)} H_{R}\left(\widetilde{G}_{i j}\right) X^{i} Y^{j} .
$$

For any $\lambda \in \kappa[X, Y]^{h m i}$, upon letting $R^{\prime}=\delta(\lambda)$ and $(z, \zeta)$ be as in Subsection (2B), by (2.3) and (2.4) we see that:

$$
\left\{\begin{array}{l}
z \in M\left(R^{\prime}\right) \backslash M\left(R^{\prime}\right)^{2} \text { with } M(R) R^{\prime}=z R^{\prime} \\
\text { and upon letting } G^{\prime}=G / z^{d(G)} \\
\text { we have }\left(R, x, y, \kappa, R^{\prime}, G R\right)(G)=G^{\prime} \in R^{\prime} \backslash z R^{\prime} \\
\text { and upon letting } d^{\prime}(G)=\operatorname{ord}_{R^{\prime}} G^{\prime} \text { with } e^{\prime}(G)=\operatorname{ord}_{\left(R^{\prime} / z R^{\prime}\right)} G^{\prime} \\
\text { we have that } d^{\prime}(G), e^{\prime}(G) \text { are nonnegative integers with } d^{\prime}(G) \leq e^{\prime}(G) \\
\text { such that } d^{\prime}(G)=0 \Leftrightarrow e^{\prime}(G)=0
\end{array}\right.
$$

and

$$
\left\{\begin{array}{l}
\text { Let } e(G) \text { be the unique nonnegative integer such that } \\
\bar{G}(X, Y) \in\left(H_{R}(\lambda)^{e(G)} K[X, Y]\right) \backslash\left(H_{R}(\lambda)^{e(G)+1} K[X, Y]\right) . \\
\text { Then } e^{\prime}(G)=e(G)
\end{array}\right.
$$

By factoring $\bar{G}$ we obtain

$$
\bar{G}(X, Y)=\widehat{G} \prod_{1 \leq i \leq h(G)} H_{R}\left(\bar{G}_{i}\right)(X, Y)^{e_{i}(G)}
$$

with

$$
\widehat{G} \in K^{\times} \text {and } \operatorname{deg}_{X, Y} H_{R}\left(\bar{G}_{i}\right)=\omega_{i}(G) \in \mathbb{N}_{+},
$$

where $h(G), e_{1}(G), \ldots, e_{h(G)}(G)$ are positive integers and $\bar{G}_{1}, \ldots, \bar{G}_{h(G)}$ are pairwise distinct members of $\kappa[X, Y]^{h m i}$. Clearly

$$
e_{1}(G) \omega_{1}(G)+\cdots+e_{h}(G) \omega_{h(G)}(G)=d(G) .
$$

For $1 \leq i \leq h(G)$ we let

$$
z_{i}(G)= \begin{cases}y & \text { if } \bar{G}_{i}=X \\ x & \text { if } \bar{G}_{i} \neq X\end{cases}
$$

with

$$
G_{i}^{\prime}=G / z_{i}(G)^{d(G)} \text { and } R_{i}^{\prime}(G)=\delta\left(\bar{G}_{i}\right)
$$

and upon letting

$$
\alpha_{i}(G): R_{i}^{\prime}(G) \rightarrow V_{i}^{\prime}(G)=R_{i}^{\prime}(G) / z_{i}(G) R_{i}^{\prime}(G)
$$

be the residue class epimorphism and observing that

$$
G_{i}^{\prime} \in R_{i}^{\prime}(G)=\text { a two-dimensional regular local domain }
$$

with

$$
V_{i}^{\prime}(G)=\text { a one-dimensional regular local domain }
$$

we let

$$
d_{i}^{\prime}(G)=\operatorname{ord}_{R_{i}^{\prime}(G)} G_{i}^{\prime} \text { and } e_{i}^{\prime}(G)=\operatorname{ord}_{V_{i}^{\prime}(G)} \alpha_{i}(G)\left(G_{i}^{\prime}\right)
$$


By $(2 \mathrm{G})$ to $(4 \mathrm{G})$ we get the following:

$(6 \mathrm{G})$

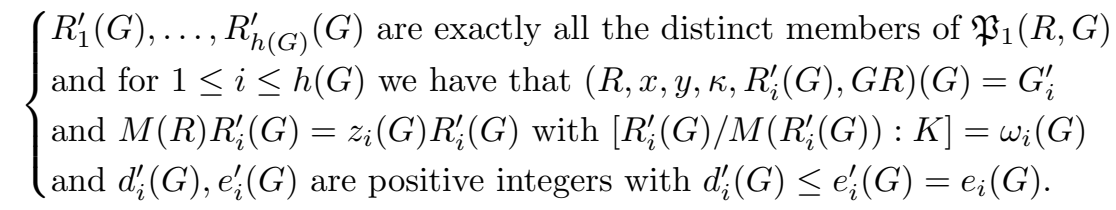

By $(5 \mathrm{G})$ and $(6 \mathrm{G})$ we see that

$$
\text { for } 1 \leq i \leq h(G) \text { we have } d_{i}^{\prime}(G) \leq d(G)
$$

and

$$
\left\{\begin{array}{l}
\text { if } e_{i}(G)=1 \text { for some } i \in\{1, \ldots, h(G)\} \\
\text { then for that } i \text { we have } M\left(R_{i}^{\prime}(G)\right)=\left(G_{i}^{\prime}, z_{i}(G)\right) R_{i}^{\prime}(G)
\end{array}\right.
$$

and

$$
\left\{\begin{array}{l}
\text { if } d_{i}^{\prime}(G) \geq d(G) \text { for some } i \in\{1, \ldots, h(G)\}, \\
\text { then } h(G)=1=\omega_{1}(G)=\left[R_{1}^{\prime}(G) / M\left(R_{1}^{\prime}(G)\right): K\right]=1 \\
\text { with } d(G)=d_{1}^{\prime}(G)=e_{1}^{\prime}(G)=e_{1}(G) .
\end{array}\right.
$$

Turning to the two-dimensional regular local domain $R^{t}$ with quotient field $L^{t}$ and maximal ideal $M\left(R^{t}\right)=(x, y) R^{t}$, we get a coefficient set $\kappa(t)$ of $R^{t}$, where $\kappa(t)$ is the set of all members of $L(t)$ which, when written in reduced form with monic denominators, has for its numerator and denominator, polynomial expressions in $t$ with coefficients in $\kappa$. Note that the residue field $H\left(R^{t}\right)$ can be identified with $K(\tau)$, where the element $\tau=H_{R^{t}}(t)$ is transcendental over $K$. For the graded ring of $R^{t}$ we have $\operatorname{grad}\left(R^{t}\right)=K(\tau)[X, Y]$. Now $\kappa(t)[X, Y]^{h m i}$ is the set of all $\lambda=\lambda(X, Y) \in \kappa(t)[X, Y]^{h m}$ such that $H_{R^{t}}(\lambda)=H_{R^{t}}(\lambda(X, Y))$ is irreducible in $K(\tau)[X, Y]$. By changing $(R, \kappa, \delta)$ to $\left(R^{t}, \kappa(t), \delta^{t}\right)$ in $(2 \mathrm{~B})(3)$ we get the tangential bijection $\delta^{t}: \kappa(t)[X, Y]^{h m i} \rightarrow Q_{1}\left(R^{t}\right)$ as described there. This also gives the QDT $\left(R^{\prime}, x^{\prime}, y^{\prime}, \kappa^{\prime}\right)$ of $\left(R^{t}, x, y, \kappa(t)\right)$ in the tangent direction $\lambda \in \kappa(t)[X, Y]^{h m i}$. Clearly

$$
\left\{\begin{array}{l}
\kappa[X, Y]^{h m i} \subset \kappa(t)[X, Y]^{h m i} \text { and } \\
\text { for all } \lambda \in \kappa[X, Y]^{h m i} \text { we have } \delta^{t}(\lambda)=\delta(\lambda)^{t}
\end{array}\right.
$$

Given any $0 \neq \Phi \in M\left(R^{t}\right)$, we can write

$$
\Phi=\sum_{i+j=d(\Phi)} \widetilde{\Phi}_{i j} x^{i} y^{j} \quad \text { with } \quad d(\Phi)=\operatorname{ord}_{R^{t}} \Phi \quad \text { and } \quad \widetilde{\Phi}_{i j} \in R
$$

and

$$
\bar{\Phi}=\bar{\Phi}(X, Y)=\operatorname{info}(\Phi)=\operatorname{info}_{\left(R^{t}, x, y\right)} \Phi=\sum_{i+j=d(\Phi)} H_{R^{t}}\left(\widetilde{\Phi}_{i j}\right) X^{i} Y^{j}
$$


For any $\lambda \in \kappa(t)[X, Y]^{h m i}$, upon letting $R^{\prime}=\delta^{t}(\lambda)$ and $(z, \zeta)$ be as in Subsection (2B), by (2.3) and (2.4) we see that:

$$
\left\{\begin{array}{l}
z \in M\left(R^{\prime}\right) \backslash M\left(R^{\prime}\right)^{2} \text { with } M\left(R^{t}\right) R^{\prime}=z R^{\prime} \\
\text { and upon letting } \Phi^{\prime}=\Phi / z^{d(\Phi)} \\
\text { we have }\left(R, x, y, \kappa(t), R^{\prime}, \Phi R^{t}\right)(\Phi)=\Phi^{\prime} \in R^{\prime} \backslash z R^{\prime} \\
\text { and upon letting } d^{\prime}(\Phi)=\operatorname{ord}_{R^{\prime}} \Phi^{\prime} \text { with } e^{\prime}(\Phi)=\operatorname{ord}_{\left(R^{\prime} / z R^{\prime}\right)} \Phi^{\prime} \\
\text { we have that } d^{\prime}(\Phi), e^{\prime}(\Phi) \text { are nonnegative integers with } d^{\prime}(\Phi) \leq e^{\prime}(\Phi) \\
\text { such that } d^{\prime}(\Phi)=0 \Leftrightarrow e^{\prime}(\Phi)=0
\end{array}\right.
$$

and

$$
\left\{\begin{array}{l}
\text { Let } e(\Phi) \text { be the unique nonnegative integer such that } \\
\bar{\Phi}(X, Y) \in\left(H_{R^{t}}(\lambda)^{e(\Phi)} K[X, Y]\right) \backslash\left(H_{R^{t}}(\lambda)^{e(\Phi)+1} K(\tau)[X, Y]\right) . \\
\text { Then } e^{\prime}(\Phi)=e(\Phi) .
\end{array}\right.
$$

By factoring $\bar{\Phi}$ we obtain

$$
\bar{\Phi}(X, Y)=\widehat{\Phi} \prod_{1 \leq i \leq h(\Phi)} H_{R^{t}}\left(\bar{\Phi}_{i}\right)(X, Y)^{e_{i}(\Phi)}
$$

with

$$
\widehat{\Phi} \in K(\tau)^{\times} \text {and } \operatorname{deg}_{X, Y} H_{R^{t}}\left(\bar{\Phi}_{i}\right)=\omega_{i}(\Phi) \in \mathbb{N}_{+},
$$

where $h(\Phi), e_{1}(\Phi), \ldots, e_{h(\Phi)}(\Phi)$ are positive integers and $\bar{\Phi}_{1}, \ldots, \bar{\Phi}_{h(\Phi)}$ are pairwise distinct members of $\kappa(t)[X, Y]^{h m i}$. Clearly

$$
e_{1}(\Phi) \omega_{1}(\Phi)+\cdots+e_{h}(\Phi) \omega_{h(\Phi)}(\Phi)=d(\Phi) .
$$

For $1 \leq i \leq h(\Phi)$ we let

with

$$
z_{i}(\Phi)= \begin{cases}y & \text { if } \bar{\Phi}_{i}=X \\ x & \text { if } \bar{\Phi}_{i} \neq X\end{cases}
$$

and upon letting

$$
\Phi_{i}^{\prime}=\Phi / z_{i}(\Phi)^{d(\Phi)} \text { and } R_{i}^{\prime}(\Phi)=\delta^{t}\left(\bar{\Phi}_{i}\right),
$$

$$
\alpha_{i}(\Phi): R_{i}^{\prime}(\Phi) \rightarrow V_{i}^{\prime}(\Phi)=R_{i}^{\prime}(\Phi) / z_{i}(\Phi) R_{i}^{\prime}(\Phi)
$$

be the residue class epimorphism and observing that

$$
\Phi_{i}^{\prime} \in R_{i}^{\prime}(\Phi)=\text { a two-dimensional regular local domain }
$$

with

$$
V_{i}^{\prime}(\Phi)=\text { a one-dimensional regular local domain }
$$

we let

$$
d_{i}^{\prime}(\Phi)=\operatorname{ord}_{R_{i}^{\prime}(\Phi)} \Phi_{i}^{\prime} \text { and } e_{i}^{\prime}(\Phi)=\operatorname{ord}_{V_{i}^{\prime}(\Phi)} \alpha_{i}(\Phi)\left(\Phi_{i}^{\prime}\right) .
$$

By $(2 \Phi)$ to $(4 \Phi)$ we get the following:

$$
\left\{\begin{array}{l}
R_{1}^{\prime}(\Phi), \ldots, R_{h(\Phi)}^{\prime}(\Phi) \text { are exactly all the distinct members of } \mathfrak{P}_{1}\left(R^{t}, \Phi\right) \\
\text { and for } 1 \leq i \leq h(\Phi) \text { we have that }\left(R^{t}, x, y, \kappa(t), R_{i}^{\prime}(\Phi), \Phi R^{t}\right)(\Phi)=\Phi_{i}^{\prime} \\
\text { and } M\left(R^{t}\right) R_{i}^{\prime}(\Phi)=z_{i}(\Phi) R_{i}^{\prime}(\Phi) \text { with }\left[R_{i}^{\prime}(\Phi) / M\left(R_{i}^{\prime}(\Phi)\right): K(\tau)\right]=\omega_{i}(\Phi) \\
\text { and } d_{i}^{\prime}(\Phi), e_{i}^{\prime}(\Phi) \text { are positive integers with } d_{i}^{\prime}(\Phi) \leq e_{i}^{\prime}(\Phi)=e_{i}(\Phi) .
\end{array}\right.
$$


By $(5 \Phi)$ and $(6 \Phi)$ we see that

$$
\text { for } 1 \leq i \leq h(\Phi) \text { we have } d_{i}^{\prime}(\Phi) \leq d(\Phi)
$$

and

$$
\left\{\begin{array}{l}
\text { if } e_{i}(\Phi)=1 \text { for some } i \in\{1, \ldots, h(\Phi)\} \\
\text { then for that } i \text { we have } M\left(R_{i}^{\prime}(\Phi)\right)=\left(\Phi_{i}^{\prime}, z_{i}(\Phi)\right) R_{i}^{\prime}(\Phi)
\end{array}\right.
$$

and

$$
\left\{\begin{array}{l}
\text { if } d_{i}^{\prime}(\Phi) \geq d(\Phi) \text { for some } i \in\{1, \ldots, h(\Phi)\} \\
\text { then } h(\Phi)=1=\omega_{1}(\Phi)=\left[R_{1}^{\prime}(\Phi) / M\left(R_{1}^{\prime}(\Phi)\right): K(\tau)\right]=1 \\
\text { with } d(\Phi)=d_{1}^{\prime}(\Phi)=e_{1}^{\prime}(\Phi)=e_{1}(\Phi) .
\end{array}\right.
$$

Henceforth assume that $\Phi=F+t G$ and recall that $J=(F, G) R$. Now clearly

$$
d, d(G), d(\Phi) \text { are positive integers with } d(\Phi)=\min (d, d(G))
$$

and

$$
\bar{\Phi}= \begin{cases}\bar{F} & \text { if } d<d(G) \\ \tau \bar{G} & \text { if } d>d(G) \\ \bar{F}+\tau \bar{G} & \text { if } d=d(G)\end{cases}
$$

Therefore we have the following three exhaustive but mutually disjoint cases:

case (E) when $d=d(G)$ with $\bar{F} / \bar{G} \notin K$;

case (F) when either $d=d(G)$ with $\bar{F} / \bar{G} \in K$ or $d<d(G)$;

case $(\mathrm{G})$ when $d>d(G)$.

Calculation (4.3). Assume case (E). Recalling the exact definition of GCD from Remark (4.0) of [Ab7] let

$$
F^{E}=\operatorname{GCD}(\bar{F}, \bar{G}) \text { with } F^{D}=\bar{F} / F^{E} \text { and } G^{D}=\bar{G} / F^{E} .
$$

Then

$$
\bar{F}=F^{E} F^{D} \text { and } \bar{G}=F^{E} G^{D},
$$

where $F^{E}, F^{D}, G^{D}$ are nonzero homogeneous polynomials in $K[X, Y]$ whose $(X, Y)$ degree is $d(E), d(D), d(D)$ respectively, where

$$
d(E) \in \mathbb{N} \text { and } d(D)=d-d(E) \in \mathbb{N}_{+} .
$$

The factorizations of $\bar{F}$ and $\bar{G}$ can be relabelled so that

$$
\left\{\begin{array}{l}
\text { for some } a, b, c, h^{*} \text { in } \mathbb{N} \text { with } a+b+c=h^{*} \leq h \\
\text { and } e_{1}^{*}, \ldots, e_{h^{*}}^{*} \text { in } \mathbb{N}_{+} \text {with } e_{1}^{*}=e_{1}=e_{1}(G), \ldots, e_{a}^{*}=e_{a}=e_{a}(G) \\
\text { and } e_{a+1}^{*}=e_{a+1}<e_{a+1}(G), \ldots, e_{a+b}^{*}=e_{a+b}<e_{a+b}(G) \\
\text { and } e_{a+b+1}^{*}=e_{a+b+1}(G)<e_{a+b+1}, \ldots, e_{a+b+c}^{*}<e_{a+b+c}(G)<e_{a+b+c}
\end{array}\right.
$$

we have

and

$$
H_{R}\left(\bar{F}_{1}\right)=H_{R}\left(\bar{G}_{1}\right), \ldots, H_{R}\left(\bar{F}_{h^{*}}\right)=H_{R}\left(\bar{G}_{h^{*}}\right)
$$

$$
F^{E}=\prod_{1 \leq i \leq h^{*}} H_{R}\left(\bar{F}_{i}\right)(X, Y)^{e_{i}^{*}}
$$

with

$$
F^{D}=\widehat{F} \prod_{a+b+1 \leq i \leq a+b+c} H_{R}\left(\bar{F}_{i}\right)(X, Y)^{e_{i}^{*}-e_{i}} \prod_{h^{*}+1 \leq i \leq h} H_{R}\left(\bar{F}_{i}(X, Y)\right)^{e_{i}}
$$


and

$$
G^{D}=\widehat{G} \prod_{a+1 \leq i \leq a+b} H_{R}\left(\bar{G}_{i}\right)(X, Y)^{e_{i}^{*}-e_{i}(G)} \prod_{h^{*}+1 \leq i \leq h} H_{R}\left(\bar{G}_{i}(X, Y)\right)^{e_{i}(G)} .
$$

For $1 \leq i \leq h^{*}$ let

$$
R_{i}^{*}=\lambda\left(\bar{F}_{i}\right) \text { with } F_{i}^{*}=F / z_{i}^{d} \text { and } G_{i}^{*}=G / z_{i}^{d} .
$$

Then in view of (2.1) and (2.3) we see that

$$
\left\{\begin{array}{l}
R_{1}^{*}, \ldots, R_{h^{*}}^{*} \text { are exactly all the distinct members of } \mathfrak{Q}_{1}(R, J) \\
\text { and for } 1 \leq i \leq h^{*} \text { we have }\left(R, x, y, \kappa, R_{i}^{*}, J\right)(F, G)=\left(F_{i}^{*}, G_{i}^{*}\right) .
\end{array}\right.
$$

We have

$$
\bar{\Phi}=F^{E}\left(F^{D}+\tau G^{D}\right)
$$

and hence $h(\Phi)=h^{*}+1$ and the factorization of $\Phi$ can be relabelled so that for $1 \leq i \leq h^{*}$ we have $\bar{\Phi}_{i}=\bar{F}_{i}$ with $e_{i}(\Phi)=e_{i}^{*}$. Now $e_{h^{*}+1}(\Phi)=1$ and there is a unique $\Phi^{D} \in \kappa(t)[X, Y]^{h m i}$ with $\widehat{\Phi} \Phi^{D}=F^{D}+t G^{D}$. By $(1 \Phi)$ to $(8 \Phi)$ we conclude that

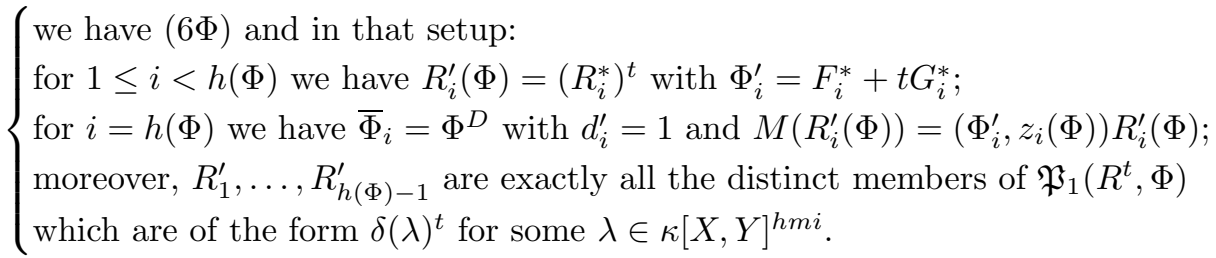

Calculation (4.4). Assume case (F). Now $d(\Phi)=d$ with $h(\Phi)=h$ and hence the factorization of $\bar{\Phi}$ can be relabelled so that for $1 \leq i \leq h$ we have $\bar{\Phi}_{i}=\bar{F}_{i}$ and $e_{i}(\Phi)=e_{i}$. For $1 \leq i \leq h$ let $R_{i}^{*}=\lambda\left(\bar{F}_{i}\right)$ with $F_{i}^{*}=F / z_{i}^{d}$ and $G_{i}^{*}=G / z_{i}^{d}$. Then in view of (2.1) and (2.3) we see that

$$
\left\{\begin{array}{l}
R_{1}^{*}, \ldots, R_{h}^{*} \text { are exactly all the distinct members of } \mathfrak{Q}_{1}(R, J) \\
\text { and for } 1 \leq i \leq h \text { we have }\left(R, x, y, \kappa, R_{i}^{*}, J\right)(F, G)=\left(F_{i}^{*}, G_{i}^{*}\right)
\end{array}\right.
$$

and by $(1 \Phi)$ to $(6 \Phi)$ we conclude that

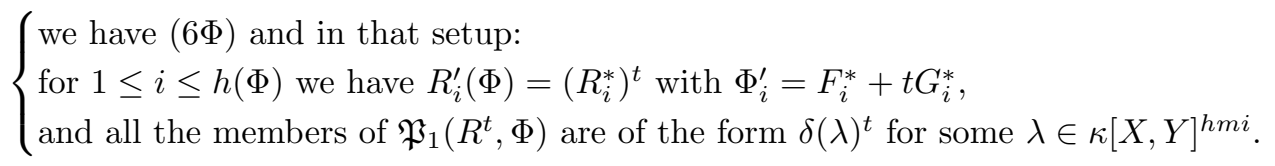

Calculation (4.5). Assume case $(\mathrm{G})$. Now $d(\Phi)=d(G)$ with $h(\Phi)=h(G)$ and hence the factorization of $\bar{\Phi}$ can be relabelled so that for $1 \leq i \leq h(G)$ we have $\bar{\Phi}_{i}=\bar{G}_{i}$ and $e_{i}(\Phi)=e_{i}(G)$. For $1 \leq i \leq h(G)$ let $R_{i}^{*}=\lambda\left(\bar{G}_{i}\right)$ with $F_{i}^{*}=F / z_{i}^{d(G)}$ and $G_{i}^{*}=G / z_{i}^{d(G)}$. Then in view of (2.1) and (3G) we see that

$$
\left\{\begin{array}{l}
R_{1}^{*}, \ldots, R_{h(G)}^{*} \text { are exactly all the distinct members of } \mathfrak{Q}_{1}(R, J) \\
\text { and for } 1 \leq i \leq h(G) \text { we have }\left(R, x, y, \kappa, R_{i}^{*}, J\right)(F, G)=\left(F_{i}^{*}, G_{i}^{*}\right)
\end{array}\right.
$$

and by $(1 \Phi)$ to $(6 \Phi)$ we conclude that

$$
\left\{\begin{array}{l}
\text { we have }(6 \Phi) \text { and in that setup: } \\
\text { for } 1 \leq i \leq h(\Phi) \text { we have } R_{i}^{\prime}(\Phi)=\left(R_{i}^{*}\right)^{t} \text { with } \Phi_{i}^{\prime}=F_{i}^{*}+t G_{i}^{*}, \\
\text { and all the members of } \mathfrak{P}_{1}\left(R^{t}, \Phi\right) \text { are of the form } \delta(\lambda)^{t} \text { for some } \lambda \in \kappa[X, Y]^{h m i} .
\end{array}\right.
$$


The above three calculations yield:

Theorem (4.6). Recall that $J=(F, G) R$ is an $M(R)$-primary ideal in $R$ and $\Phi=$ $F+t G$. Let $R_{1}^{*}, \ldots, R_{h^{*}}^{*}$ be all the distinct members of $\mathfrak{Q}_{1}(R, J)$ and for $1 \leq i \leq h^{*}$ let $\lambda_{i}^{*}=\delta^{-1}\left(R_{i}^{*}\right)$ and $R_{i}^{\prime}=\left(R_{i}^{*}\right)^{t}$ and $\left(F_{i}^{*}, G_{i}^{*}\right)=\left(R, x, y, \kappa, R_{i}^{*}, J\right)(F, G)$. Then we have the following.

(4.6.1) $R_{1}^{\prime}, \ldots, R_{h^{*}}^{\prime}$ are exactly all those distinct members of $\mathfrak{P}_{1}\left(R^{t}, \Phi\right)$ which are of the form $\delta(\lambda)^{t}$ for some $\lambda \in \kappa[X, Y]^{h m i}$. $\lambda_{1}^{*}, \ldots, \lambda_{h^{*}}^{*}$ are exactly all the distinct tangent directions of $\Phi$ in $\kappa[X, Y]^{h m i}$. For $1 \leq i \leq h^{*}$ we have $\left(R^{t}, x, y, \kappa(t), R_{i}^{\prime}\right.$, $\left.\Phi R^{t}\right)(\Phi)=F_{i}^{*}+t G_{i}^{*}$ and $\delta^{t}\left(\lambda_{i}^{*}\right)=R_{i}^{\prime}$.

(4.6.2) If either $\operatorname{ord}_{R} F \neq \operatorname{ord}_{R} G$ or $\operatorname{info}(F) / \operatorname{info}(G) \in K$, then $R_{1}^{\prime}, \ldots, R_{h^{*}}^{\prime}$ are exactly all the distinct members of $\mathfrak{P}_{1}\left(R^{t}, \Phi\right)$.

(4.6.3) If $\operatorname{ord}_{R} F=\operatorname{ord}_{R} G$ and $\operatorname{info}(F) / \operatorname{info}(G) \notin K$, then there is exactly one member $R^{\prime}$ of $\mathfrak{P}_{1}\left(R^{t}, \Phi\right)$ different from $R_{1}^{\prime}, \ldots, R_{h^{*}}^{\prime}$. Moreover, upon letting $\Phi^{\prime}=\left(R^{t}, x, y, \kappa(t), R^{\prime}, \Phi R^{t}\right)(\Phi)$ we have $\operatorname{ord}_{R^{\prime}} \Phi^{\prime}=1$. More precisely, upon letting $d=\operatorname{ord}_{R} F$ we have $\Phi^{\prime}=\Phi / x^{d}$ with $M\left(R^{\prime}\right)=\left(\Phi^{\prime}, x\right) R^{\prime}$, and upon letting $\Phi^{D}=\left(\delta^{t}\right)^{-1}\left(R^{\prime}\right)$ we have

$$
\bar{\Phi}=\widehat{\Phi} H_{R^{t}}\left(\Phi^{D}\right) \prod_{1 \leq i \leq h^{*}} H_{R}\left(\lambda_{i}^{*}\right)^{e_{i}^{*}} \text { with } \widehat{\Phi} \in K(\tau)^{\times} \text {and } e_{i}^{*} \in \mathbb{N}_{+} .
$$

Note (4.7). Elsewhere, as a consequence of the above theorem, we shall show that $\left(R^{t}, \Phi R^{t}\right)$ is strongly square free.

\section{REFERENCES}

[Ab1] S. S. Abhyankar, On the valuations centered in a local domain, American Journal of Mathematics, vol. 78 (1956), pages 321-348. MR0082477(18:556b)

[Ab2] S. S. Abhyankar, Ramification Theoretic Methods in Algebraic Geometry, Princeton University Press, 1959. MR0105416 (21:4158)

[Ab3] S. S. Abhyankar, Resolution of Singularities of Embedded Algebraic Surfaces, First Edition of 1966, Published by Academic Press; Second Enlarged Edition of 1998, Published by Springer-Verlag. MR:1617523 (99c:14021)

[Ab4] S. S. Abhyankar, Historical ramblings in algebraic geometry and related algebra, American Mathematical Monthly, vol. 83 (1976), pages 409-448. MR0401754 (53:5581)

[Ab5] S. S. Abhyankar, Desingularization of plane curves, Singularities, Proceedings of Symposia in Pure Mathematics, American Mathematical Society, vol. 40, Part 1 (1983), pages 1-45. MR713043 (85d:14024)

[Ab6] S. S. Abhyankar, Algebraic Geometry for Scientists and Engineers, American Mathematical Society, 1990. MR.1075991 (92a:14001)

[Ab7] S. S. Abhyankar, Polynomial expansion, Proceedings of the American Mathematical Society, vol. 126 (1998), pages 1583-1596. MR1443142 (98g:12003)

[Ab8] S. S. Abhyankar, Lectures on Algebra I, World Scientific, 2006. MR2253320(2007k:13001)

[Ab9] S. S. Abhyankar, Inversion and Invariance of Characteristic Terms Part I, The Legacy of Alladi Ramakrishnan in the Mathematical Sciences (2010), pages 93-168, Springer. MR2744259 (2012b:14057)

[Ab10] S. S. Abhyankar, Dicritical divisors and Jacobian problem, Indian Journal of Pure and Applied Mathematics, vol. 41 (2010), pages 77-97. MR2650101 (2011d:14107)

[Ab11] S. S. Abhyankar, Pillars and towers of quadratic transformations, Proceedings of the American Mathematical Society, vol. 139 (2011), pages 3067-3082. MR2811263

[Ab12] S. S. Abhyankar, More about dicriticals, Proceedings of the American Mathematical Society, vol. 139 (2011), pages 3083-3097. MR2811264

[Nag] M. Nagata, Local Rings, Wiley, 1962. MR0155856 (27:5790)

Department of Mathematics, Purdue University, West Lafayette, Indiana 47907

E-mail address: ram@cs.purdue.edu 